JOURNAL OF

APPLIED

CRYSTALLOGRAPHY

ISSN 1600-5767

\section{Silver Nanoparticles. From Silver Halide Photo- graphy to Plasmonics. By Tadaaki Tani. Oxford University Press, 2015. Pp. 224. Price (hardcover) GBP 65.00. ISBN 978-0-19-871460-6.}

\author{
Steven J. Oldenburg* \\ nanoComposix Inc., 4878 Ronson Court, Suite K, San Diego, CA 92111, USA. *Correspondence e-mail: \\ steve.oldenburg@nanocomposix.com
}

Tadaaki Tani's book on silver nanoparticles draws upon his nearly 50 years of experience in working with silver halide compounds in the photographic industry. The central premise of the book is that lessons learned in silver based photographic science can provide new insights and strategies for the preparation of plasmonic silver nanoparticles that are being utilized in many nanotechnology products. The work begins with an indepth description of the crystallographic structure of both silver (Ag) and silver halide structures $(\operatorname{Ag} X)$. Crystalline defects and molecular interactions with specific crystal planes provide a basis for understanding the anisotropic growth of silver nanoparticles in solution. There is a good overview of the mechanism of formation and strategies to control $\operatorname{Ag} X$ nanoparticle shape and size in thin films. Size and shape effects are summarized in a chapter on plasmonics, which explains the unusually strong interaction of small silver particles with specific wavelengths of light. Other chapters review the potential for silver nanoparticles to be used in catalysis and photovoltaic effects.

While it is clear that the author has a strong background in silver nanoparticles used for photographic applications, the complementary knowledge of silver nanoparticle fabrication and use for nanotechnology applications is missing. There is no detail provided for standard methods of fabrication, processing, concentrating or surface functionalization of silver nanomaterials or the challenges associated with aggregation and their subsequent effects on the silver nanoparticles' optical properties. Discussions on stability are primarily limited to thin films, leaving out important topics of instability in colloidal solutions due to steric/charge based stability, size-dependent dissolution and effects such as Ostwald ripening. The background description of plasmon resonance provides a good introduction, but more information on the optical properties as they relate to the specifics of scattering/absorption ratios as a function of size and the extinction efficiencies would have better communicated how unusual the properties of silver nanoparticles are, and why their use as ultra-bright labels for molecular sensing, novel optical filters and targeted photothermal therapies is so promising. Consideration of the silver dissolution rate as it relates to providing broad based antimicrobial function would also have been of interest as this is the largest commercial use of silver nanoparticles. Discussions on depressed melting points as they relate to silver nanoparticles' use in conductive inks would have fitted well into sections that described size-dependent stability.

Chapter 7 on the stability of Ag and metal nanoparticles communicated the author's desire to leverage the knowledge that had been acquired via decades of photographic research into assisting nanoparticle researchers with the ongoing challenge of stability. A detailed description of the mechanism by which gelatin provides multi-year stability in photographic paper is provided, and it is suggested that this compound be further investigated as a coating for silver nanoparticles. The data provided here are useful and may be helpful as a basis for investigations into gelatin based coatings. However, there are many requirements for the selection of the optimal surface beside long-term resistance to sulfidation, and factors such as the thickness of the protective layer, the layer's stability with time when incorporated into other matrices, and the ability to further functionalize the surface with targeting ligands or other functional coatings must be considered. 


\section{book reviews}

Overall this book provides a valuable resource for summarizing silver and silver halide based nanoparticles. It is important that researchers who are fabricating silver nanoparticles are aware of the long history and use of this class of materials in the photographic industry and try to learn as much as possible from the available data. As a starting place for acquiring this background, this book is a good resource; for understanding the challenges, strategies and ultimate applications of emerging applications of silver nanoparticles readers will have to look elsewhere. 